\title{
Apprentissage par projet autour d'un robot mobile : partage d'expériences entre l'Europe et le Maroc.
}

\author{
Damien Grenier ${ }^{1}$, Saïd Belkouch ${ }^{2}$, Khalid Faitah ${ }^{2}$, Driss Yousfi ${ }^{2}$, Bruno Dehez ${ }^{3}$, \\ Laurent De Vroey ${ }^{3}$, Christophe Vloebergh ${ }^{3}$, Eric Richard ${ }^{4}$ \\ ${ }^{1}$ Ecole Normale Supérieure de Cachan, Département de Mécatronique, \\ Université Européenne de Bretagne, Campus de Ker Lann, F35170 Bruz, France \\ ${ }^{2}$ Ecole Nationale des Sciences Appliquées de Marrakech, \\ B.P 575 Av Abdelkarim Khattabi, Gueliz, Marrakech, Maroc \\ ${ }^{3}$ Université catholique de Louvain, Laboratoire d'Electrotechnique et d'Instrumentation, \\ Place du Levant 3, B1348 Louvain-La-Neuve, Belgique \\ ${ }^{4}$ ISEN-Toulon, Place G. Pompidou, F83000 Toulon, France
}

\begin{abstract}
RESUME : Cet article présente un exemple de collaboration nord-sud pour le lancement au Maroc d'une nouvelle filière de formation d'ingénieur en génie électrique. Pour permettre aux étudiants d'acquérir un sens pratique de la discipline, il a été décidé de mettre en œuvre un apprentissage par projet, en adaptant un dispositif déployé en Belgique autour de robots mobiles. De nouvelles plates-formes robotiques ont été conçues et livrées. Elles servent de support dans un premier temps à des travaux pratiques sur les microprocesseurs, les capteurs et le conditionnement de signal associé, l'électronique de puissance et la commande de machine. Dans un second temps ces connaissances sont intégrées par les étudiants dans un projet visant à faire accomplir une tâche donnée aux robots.
\end{abstract}

Mots clefs : apprentissage par projet, électronique, électronique de puissance, microcontrôleurs

\section{LE CONTEXTE}

L'Ecole Nationale des Sciences Appliquées (ENSA) de Marrakech, composante de l'Université Cady Ayyad, a été créée en 2001 sous l'impulsion de son directeur, le Pr. Mustapha El Adnani. Elle fait partie du réseau des ENSA également implanté à Agadir, Fès, Oujda, Safi et Tanger.

Elle recrute sur concours au niveau bac et forme des Ingénieurs d'Etat en 5 ans dont 3 ans de tronc commun et 2 années de spécialisation, soit en Génie Informatique, soit en Génie des Réseaux et Télécommunications, soit en Génie Electrique, soit encore, prochainement, en Génie Industriel.

Pour la mise en place des filières de spécialisation, l'ENSAM a bénéficié à partir de la rentrée 2003 du soutien d'un consortium franco-belge d'écoles d'ingénieur et d'université, coordonné par l'Ecole Centrale de Marseille, dans le cadre d'un des tous premiers projets TEMPUS-MEDA, financé par la commission européenne.

$\mathrm{Au}$ sein de ce consortium, l'ISEN-Toulon était plus particulièrement impliqué dans la mise en place des filières Génie Informatique et Génie des Réseaux et Télécommunications qui devaient ouvrir dès 2004 (et donc diplômer ses premiers étudiants en 2006). L'Université catholique de Louvain ${ }^{1}$ devait, elle, aider à la création de la filière Génie Electrique dont l'ouverture était retardée d'un an.

\footnotetext{
${ }^{1}$ Le coordinateur du projet pour l'UCL, a, dans la dernière année du projet, rejoint l'ENS de Cachan où il a continué à superviser l'aide à la mise en place de la formation.
}

Pour cette filière Génie Electrique, il avait été imaginé, dans le projet initial, de mettre en place un dispositif pédagogique associant étroitement, pour la partie pratique de la formation, des laboratoires réels s'appuyant sur du matériel didactique financé par le Ministère Marocain de l'Enseignement Supérieur, complétés par des laboratoires virtuels utilisant des outils multimédia et de simulations (laboratoires virtuels) dans le développement desquels l'UCL dispose d'une solide expérience (voir par exemple [1]).

Nous avons cependant mis a profit le délai que nous offrait l'ouverture décalée de la filière Génie Electrique pour revoir cette option. Nous avons ainsi pris le temps d'analyser le contexte socio-économique de la région de Marrakech (dimension régionale de l'ENSAM au sein du réseau des ENSA et des autres écoles d'ingénieurs marocaines oblige) où l'activité économique repose principalement sur l'agriculture et l'agroalimentaire d'une part, et les services d'autre part (au premier rang desquels évidemment, le tourisme). L'activité industrielle y est relativement limitée, animée essentiellement par de petites et moyennes entreprises. Cette situation risque de perdurer, un développement industriel massif de Marrakech étant difficilement compatible avec le maintien du potentiel touristique de la région.

Les débouchés régionaux éventuels pour la formation d'ingénieurs en Génie Electrique correspondent donc à des profils d'ingénieurs relativement polyvalents, capables de comprendre, choisir, régler, maintenir tous les éléments d'une chaîne de conversion électrique, du système de distribution aux systèmes de commande des actionneurs impliqués dans un 
processus de fabrication. Cela suppose surtout le développement d'un sens pratique qu'un enseignement de masse avec des équipements matériels réduits ne leur a pas permis d'acquérir avant leur entrée à l'Ecole ni même au cours des premières années de tronc commun qui s'appuient beaucoup sur les moyens de l'université et notamment de la Faculté des Sciences et Techniques (FST) voisine.

Développer un dispositif pédagogique faisant une large part à des expérimentations virtuelles, risquait, dans ces conditions, ne nous faire passer complètement à côté de l'objectif de formation de "praticiens". Il a donc été rapidement décidé de déployer un tout autre dispositif pédagogique, en se basant sur une autre compétence développée par l'UCL pour ses propres étudiants, à savoir la pédagogie par projet.

Depuis plus de 10 ans, l'UCL organise en effet, dans le cadre de la filière de formation en électromécanique, orientation mécatronique, un enseignement par projet intégrant les aspects liés à la conception mécanique, l'actionnement (électronique de puissance et commande des moteurs), l'électronique (capteurs et traitement des signaux associés, électronique numérique de commande) et l'informatique temps réel. Ce projet, appelé projet intégré en mécatronique, mobilise $1 / 3$ du volume horaire de la $4^{\text {ème }}$ année de formation, autour d'un objectif commun, à savoir la conception et la réalisation d'un robot mobile [2], [3], devant participer à une compétition initialement interne, puis à partir de 2005 externe à l'université (Coupe de Belgique de Robotique).

C'est ce type d'expérience qui a donc finalement été décidé de tenter de transférer de la Belgique vers le Maroc, en tentant de garder, à tout instant, une approche pragmatique, adaptée aux moyens humains et matériels locaux.

Cet article décrit le matériel pédagogique développé par l'UCL et l'ISEN-Toulon (pour la partie informatique temps réel) au profit de l'ENSAM. Il montre ensuite comment les enseignants marocains se sont appropriés ces dispositifs, comment ils les ont intégrés dans leur dispositif pédagogique et quel impact cela a eu sur la formation des ingénieurs en Génie Electrique de l'ENSAM.

\section{LES PLATES-FORMES ROBOTIQUES}

Dans le cadre du projet intégré en mécatronique organisé à l'UCL, les étudiants sont amenés à concevoir et réaliser (avec un éventuel support technique des techniciens des départements impliqués) un robot mobile capable de participer à une compétition (et si possible la gagner!) dont les règles changent d'année en année [3]. Cela inclut la conception et la fabrication du châssis mécanique des robots, ainsi que d'un certain nombre de cartes électroniques pour la génération des différentes tensions d'alimentations générées à partir de batteries embarquées, le traitement des signaux issus des capteurs ou encore l'alimentation des moteurs (réalisation de hacheurs pour le pilotage des moteurs à courant continu, d'onduleurs pour celui des moteurs de type "brushless").

Pour que chaque année les étudiants aient à concevoir quelque chose de nouveau et éviter que le projet ne devienne au fil des ans qu'une simple mise en œuvre par les étudiants de solutions déjà développées par leurs prédécesseurs, certaines fonctions leur sont fournies sous forme de circuits imprimés dédiés (dont éventuellement certains éléments restent à dimensionner en fonction de leurs contraintes propres). De nouveaux défis leur sont par ailleurs posés, permettant ainsi au fil des années une amélioration constante des performances atteintes par les robots.

Dans le cadre de la formation en Génie Electrique de l'ENSAM, les étudiants marocains n'ont, contrairement à leurs homologues belges, pas vocation à concevoir la structure mécanique des robots.

Trois types de plates-formes ont donc été développées à l'UCL et livrées à l'ENSAM (cf § 2.1) : deux platesformes bénéficiant d'une motorisation par deux roues motrices actionnées par deux moteurs (tous deux, soit de type moteur à courant continu, soit de type "brushless") et une plate-forme de type tricycle avec roue motrice et directrice à l'avant.

Pour permettre une certaine modularité des platesformes, un système de racks a été développé pour abriter les cartes électroniques de puissance (alimentation, hacheurs, onduleurs, cf § 2.3), ainsi que les cartes de traitement de signal des capteurs impliqués (cf. §2.4).

La commande du robot devait être réalisée au moyen d'un microprocesseur ST7 de ST Microelectronics, grâce à une carte de commande développée par l'ISEN-Toulon (cf. §2.5). Pour la partie contrôle, nous avons en effet choisi de ne pas utiliser les solutions habituellement mises en œuvre à l'UCL, afin de ne pas multiplier inutilement les familles de microprocesseurs étudiées à l'ENSAM. La famille ST7 était en effet déjà utilisée dans le cadre de la formation en Génie Informatique mise en place, on le rappelle, avec le soutien de l'ISEN-Toulon.

La livraison des différents éléments a été chaque fois accompagnée de formations / dialogue d'une à deux semaine chaque, entre leurs concepteurs européens et les futurs utilisateurs marocains.

\subsection{STRUCTURES MECANIQUES DES PLATES-FORMES}

Aux trois types de plate-forme ne correspondent en réalité que deux types de structures mécaniques: 
l'une entraînée par deux roues motrices et l'autre par une seule roue à la fois motrice et directrice (Fig. 1). Dans les deux cas, l'idée était de développer une structure modulaire sur base d'un châssis de forme circulaire.

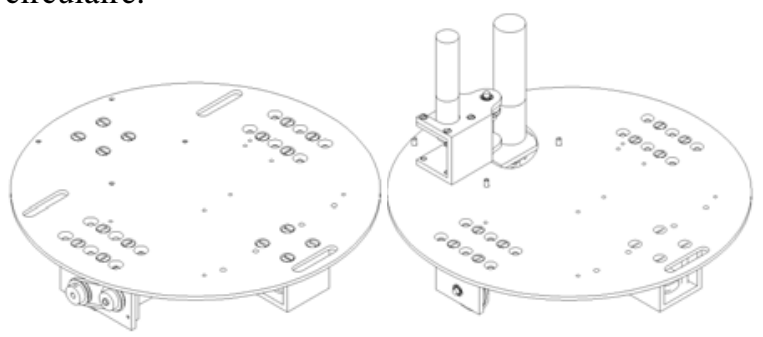

Fig. 1. Illustration des deux structures mécaniques développées dans le cadre du projet

Pour la structure à deux roues motrices, deux modules différents se fixent sur le châssis : les modules « roue motrice » servant à l'entraînement sont placés latéralement et les modules «bille porteuse » servant à la stabilisation de la plate-forme sont placés à l'avant et à l'arrière de la plate-forme (Fig. 2).

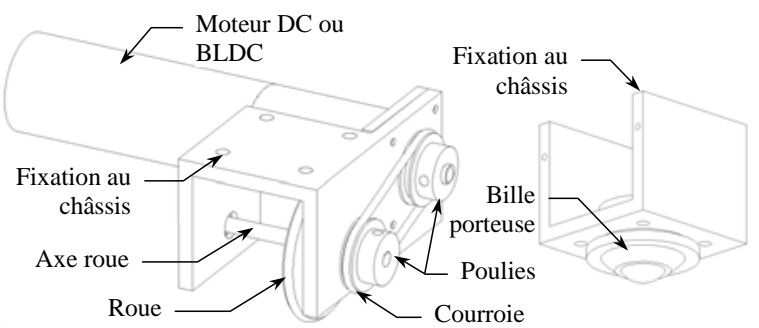

Fig. 2. Modules de la structure deux roues motrices

Pour la structure à roue motrice-directrice, trois modules différents sont nécessaires : le module « roue motrice-directrice » servant à l'entraînement est placé devant, les modules «roue passive» servant au guidage sont placés sur les cotés et le module «bille porteuse » servant à la stabilisation de la plate-forme à l'arrière (Fig. 3).

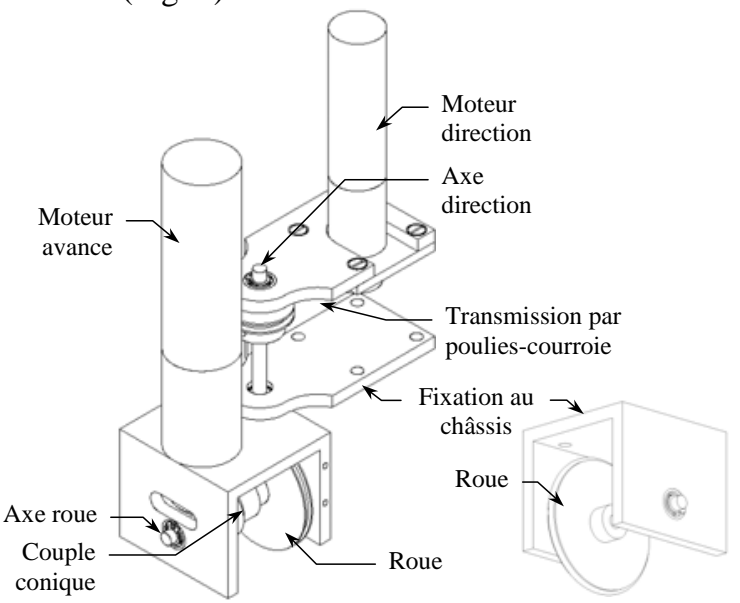

Fig. 3. Modules de la structure roue motrice - directrice

Les différents modules ont été construits de manière aussi robuste que possible afin qu'ils puissent être manipulés par les étudiants sans risque de casse.
Pour les deux structures, la fixation des modules sur le châssis est pensée de manière à faciliter le montage et le démontage des différents modules. Leurs positions relatives sont choisies de manière à permettre aux plateformes une rotation sur ellesmêmes.

\subsection{CAPTEURS ET TRAITEMENT DE SIGNAUX INTEGRES}

Afin de garantir l'autonomie fonctionnelle des platesformes, un travail spécifique a été mené dans le domaine des capteurs de position.

Sur base des contraintes et souhaits de l'ENSAM et selon l'expérience qu'en avait l'UCL, plusieurs lignes directrices ont guidé ce travail.

Pour des raisons pédagogiques, il était utile d'aborder différents types de capteurs et le traitement de signal associé à chacun d'eux. C'est ainsi que le choix s'est porté sur des capteurs optiques, électromagnétiques et laser.

Les deux premiers ont été utilisés dans un contexte de suivi de ligne au sol (détection de ligne blanches sur fond noir ou réciproquement, détection du champ magnétique créé par le courant circulant dans un fil situé sous la piste). L'amplitude du signal varie avec la distance entre le capteur et le bord de la ligne blanche ou le fil. Deux capteurs situés à quelques millimètres l'un de l'autre permettent d'identifier si l'on se situe à droite ou à gauche de la ligne ou du fil. Le capteur laser est en fait un binôme émetteur laser / phototransistor de réception, monté sur une tourelle mobile (Fig. 4). Il détecte ou non la présence dans son alignement d'un élément réfléchissant (bande catadioptre). Il renvoie donc un signal logique.

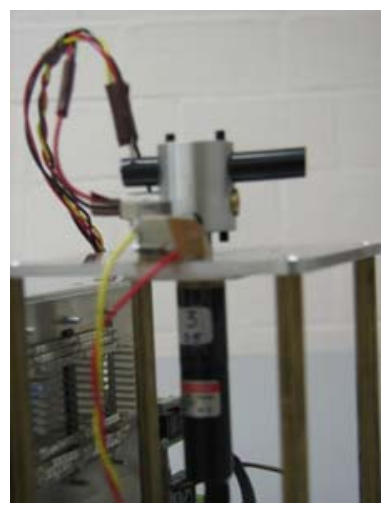

Fig. 4. Tourelle laser

Pour éviter les perturbations extérieures, il est possible pour chaque catégorie de capteur, de moduler le signal (alimentation des diodes optiques ou laser ou encore des fils situés sous la piste par un signal de fréquence donnée). On s'affranchit alors, dans une certaine mesure des variations d'éclairage ambiant ou des perturbations électromagnétiques. 


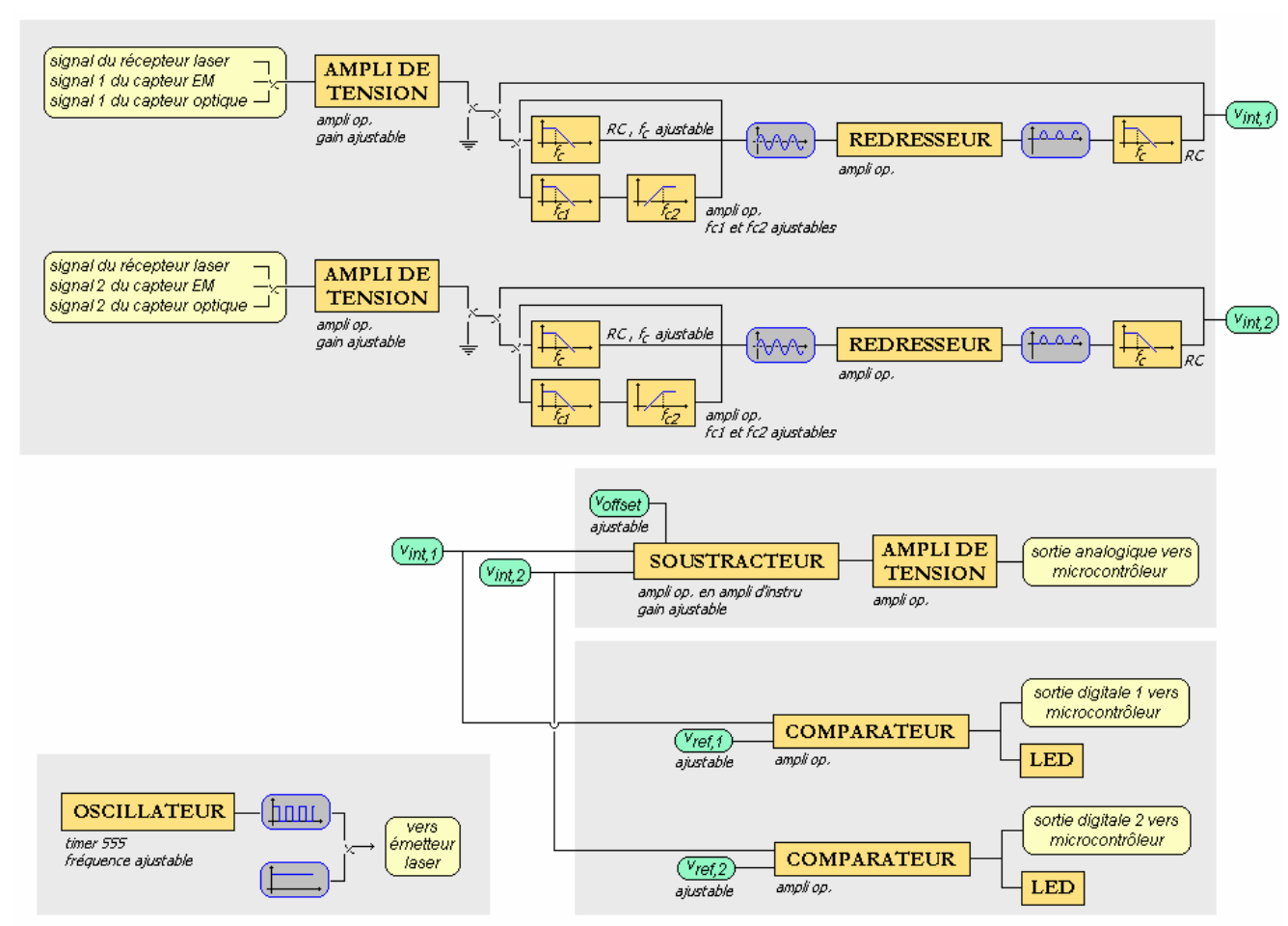

Fig. 5. Synoptique carte capteur

Dans un but d'économie pour le développement et l'entretien du matériel, il était souhaitable de limiter le nombre de cartes d'électronique de traitement. Nous avons réussi à réaliser une carte unique et modulaire, intégrant l'électronique de traitement des trois types de capteurs. Cette carte comporte différents étages classiques de traitement de signal, basés principalement sur des amplificateurs opérationnels. On y retrouve des amplificateurs de tension, filtres, redresseurs, sommateurs,... Ces étages peuvent être combinés de différentes manières, selon les capteurs utilisés et les souhaits des utilisateurs (Fig. 5).

L'ENSAM souhaitait également pouvoir utiliser les capteurs et les cartes de traitement indépendamment des plates-formes, dans le cadre de travaux pratiques spécifiques (voir 3.2). Pour cette raison, les cartes ont été réalisées avec de nombreuses possibilités d'adaptations (changement possible des valeurs de certains composants passifs stratégiques, utilisation de potentiomètres pour fixer gains et offsets,...). Des notices d'utilisation, présentées sous la forme d'énoncés de travaux pratiques, ont également été réalisées, mises en œuvre à l’UCL, puis proposées à l'ENSAM.

\subsection{ELECTRONIQUE DE PUISSANCE}

Les plates-formes robotiques sont alimentées par 6 batteries $\mathrm{Ni}-\mathrm{Cd}$ disposées en série et fournissant une tension totale de $7.2 \mathrm{~V}$. Une carte d'alimentation a été développée afin de fournir les différentes tensions nécessaires à l'alimentation des moteurs, de leurs circuits de commande ainsi que l'alimentation de tous les circuits électroniques analogiques et digitaux embarqués sur les robots (circuits de conditionnement des signaux venant des capteurs, carte microprocesseur...). La carte d'alimentation est composée d'un régulateur $5 \mathrm{~V}$ pour alimenter les circuits électroniques digitaux, d'un convertisseur flyback régulé capable de fournir une tension de +15 et $-15 \mathrm{~V}$ pour alimenter les circuits de traitement des signaux analogiques et d'un convertisseur boost non réversible $12 \mathrm{~V}$ régulé pour alimenter les moteurs DC de faible puissance $(1.5 \mathrm{~W}$ et $4.5 \mathrm{~W})$ utilisés pour orienter le laser monté sur la tourelle ou pour contrôler la direction du tricycle.

A coté de cette carte d'alimentation, nous avons réalisé, sur une carte comprenant un convertisseur boost réversible $24 \mathrm{~V}$ capable d'alimenter des moteurs de plus grande puissance (moteurs DC et "brushless" 20 et $40 \mathrm{~W}$ ) utilisés pour la propulsion des robots.

Chaque moteur DC équipant la plate-forme mobile est alimenté par un hacheur quatre quadrants piloté par un signal MLI (modulation à largeur d'impulsion) à $100 \mathrm{kHz}$ fourni, par exemple, par le microcontrôleur. Les moteurs "brushless" eux sont alimentés par un onduleur de tension. Pour des raisons d'encombrement et de modularité, la commande de ces moteurs est réalisée sur une carte spécialement dédiée à cet effet, comprenant un circuit programmable qui contient la logique de commutation nécessaire au pilotage de ces moteurs, soit par une commande en pleine onde, soit par une commande MLI. 
Lors de la conception des cartes électroniques de puissance, une attention particulière a été portée sur leur modularité non seulement au sein du robot, puisqu'elle peuvent être interchangées dans le rack qui les accueille, mais aussi en vue d'autres applications de plus grande puissance, par exemple, dans le cadre de laboratoires voire de travaux de recherche à l'ENSAM. En effet, toutes les cartes électroniques sont de taille identiques et ont été dessinées à l'aide de la version étudiante du logiciel EAGLE (disponible gratuitement), leurs schémas et prints ont été fournis aux enseignants de l'ENSAM afin que leurs étudiants puissent en comprendre le fonctionnement et les modifier en fonction d'autres besoins, notamment pour des laboratoires ou des travaux de recherche dans le cadre de le travail de fin d'études.

\subsection{CARTE MICROPROCESSEUR}

L'ISEN-Toulon, en tant qu'école généraliste formant des ingénieurs en hautes technologies, a inclus depuis longtemps la formation sur microcontrôleurs dans sa pédagogie. Celle-ci se base sur des cours magistraux complétés par un mini-projet en binôme de réalisation d'applications simples mettant en oeuvre le $\mu$ contrôleur (cœur CPU et périphériques). A cet effet, l'ISEN a développé un kit pédagogique, en collaboration avec ST Microelectronics qui comprend une carte avec un $\mu$ contrôleur ST7, différents périphériques (afficheurs, LEDs, boutons poussoirs, mini moteur, buzzer, ..) et un connecteur qui permet d'accéder à toutes les entrées sorties du $\mu$ contrôleur pour s'interfacer à de l'électronique externe. Ce kit comprend également les moyens de téléchargement et mise au point depuis un PC et un support pédagogique papier et bientôt en ligne (approche elearning).

Une quinzaine de kits a été fournie à l'ENSAM pour l'informatique embarquée. La carte à base de ST7 a été montée dans les robots de l'UCL et interfacée aux capteurs et à l'électronique de commandes des moteurs. Les étudiants peuvent ainsi programmer le robot en flashant leur code dans le ST7 depuis un PC du laboratoire. Ils mettent ainsi en pratique leurs cours de programmation embarquée appliquée à la robotique.

\section{DISPOSITIF PEDAGOGIQUE}

L'équipe marocaine a choisi d'introduire au niveau de la $3^{\text {eme }}$ année (première année du cycle d’ingénieur) les différents types de cartes (microcontôleur, capteur, électronique de puissance) comme support de travaux pratiques pour les cours correspondants. Cela permet de familiariser très tôt les étudiants avec les cartes électroniques de leur futur robot et de connaître le rôle de chaque composant sur la carte.
C'est au niveau de la $4^{\text {ème }}$ année, que toutes ces compétences sont fédérées à travers une formation par projet.

\subsection{TRAVAUX PRATIQUES SUR LES MICROPROCESSEURS}

Les travaux pratiques sur les microprocesseurs / microcontrôleurs sont données en $3^{\text {ème }}$ année comme support au cours de l'architecture des ordinateurs. Dans ce module de $90 \mathrm{~h}$, il y a $40 \mathrm{~h}$ de travaux pratiques dont $12 \mathrm{~h}$ de TP d'électronique numérique, $20 \mathrm{~h}$ en microprocesseurs et $8 \mathrm{~h}$ en organisation des ordinateurs.

Pour la partie microprocesseur, l'ENSAM utilise la carte microcontrôleur ST7 développée par l'ISENToulon. La moitié des travaux pratiques se fait en simulation en assembleur. La deuxième partie se fait sur carte. Les étudiants allument des leds au travers des ports parallèles ou allument les afficheurs 7 segments en utilisant cette fois-ci un port en série du ST7. Dans d'autres travaux pratiques, ils utilisent les interruptions pour démarrer ou arrêter une routine. Les étudiants apprennent comment exploiter le convertisseur $\mathrm{A} / \mathrm{D}$ pour générer les équivalents numériques des grandeurs physiques continues externes, faire l'analyse des données et prendre des décisions sur les actions à prendre, comme par exemple la génération d'un signal MLI pour la commande d'un moteur.

À la fin de ces TPs, l'étudiant sort avec une compréhension claire sur le fonctionnement du microprocesseur/ microcontrôleur. Il acquis des connaissances sur le rôle de chaque bloc interne au microcontrôleur. Il est prêt à passer à l'étape suivante, à savoir l'intégration de la carte microcontrôleur dans des systèmes plus complexes.

\subsection{TRAVAUX PRATIQUES SUR LES CAPTEURS}

En $3^{\text {ème }}$ année, la carte capteur du projet avec son module électronique de conditionnement a été utilisée pour faire assimiler aux étudiants le traitement de signal en général.

Ont ainsi pu être vus au cours de différents travaux pratiques

- le filtrage actif passe-bas, passe-haut et le passebande.

- le redressement de précision à base d'amplificateurs opérationnels

- le réglage d’offset dans les amplificateurs d'instrumentations

C'est en $4^{\text {ème }}$ année, que ces concepts ont réutilisés pour traiter l'information issue des capteurs situés sur les plates-formes robotiques et permettre ainsi au robot de se repérer dans l'espace ou détecter un obstacle. 


\subsection{TRAVAUX PRATIQUES EN ELECTRO- NIQUE DE PUISSANCE ET COMMANDE DES MOTEURS}

Pour mener à bien les travaux expérimentaux associés aux domaines de l'électronique de puissance et de la commande des moteurs, les enseignants marocains ont réalisé, dans un premier temps, une adaptation entre les cartes de puissances fournies avec le robot et les plates-formes DSP 'dSpace DS1104' que possède ENSAM.

Les travaux pratiques (au premier semestre de la $4^{\text {ème }}$ année) sont articulés en deux étapes :

- une étape de simulation dans l'environnement Matlab/Simulink des différentes structures envisagées dont le but est, d'une part, l'analyse de leur comportement, et d'autre part, le dimensionnement des composants ou des régulateurs mis en jeu.

- une étape d'implantation de la commande en utilisant les cartes de puissance des robots pilotées à partir de plate-forme DSP et son interface graphique ControlDesk.

Les sujets qui ont pu être traité de cette manière sont :

- la cellule de commutation dans les convertisseurs électroniques de puissance et les considérations pratiques autour de sa mise en œuvre application à un hacheur parallèle.

- l'onduleur triphasé avec MLI sinusoïdale

- le hacheur 4 quadrants et la commande d'un moteur DC

- la commande d'un moteur "brushless"

Il est à signaler que l'ENSA de Marrakech est ainsi la première école d'ingénieurs au Maroc, qui a introduit dans sa formation l'enseignement expérimental du moteur "brushless" et de sa commande.

\subsection{UTILISATION DES PLATES-FORMES ROBOTIQUES}

L'enseignement par projet a été initié le deuxième semestre de l'année scolaire 2006-2007 et il lui a été réservé un demi module. Il a été décidé pour cette première expérience de se limiter à un groupe de quatre étudiants pour faire réaliser au robot un "simple" suivi de ligne. Les autres groupes d'étudiants ont pris des projets différents. Nous comptons l'année prochaine augmenter la taille de l'enseignement par projet à un module complet. A terme (quand la formation en "génie électrique / systèmes embarqués" aura son effectif complet), 24 étudiants travailleront par groupe de 4 sur les 6 plates-formes livrées à l'ENSAM, permettant ainsi d'explorer l'ensemble des variantes.

Pour faire face à la complexité croissante des tâches à faire accomplir par les robots, le développement de cartes électroniques complémentaires permettant d'associer aux microprocesseurs ST7 des FPGA/CPLD est en cours de développement au Maroc.

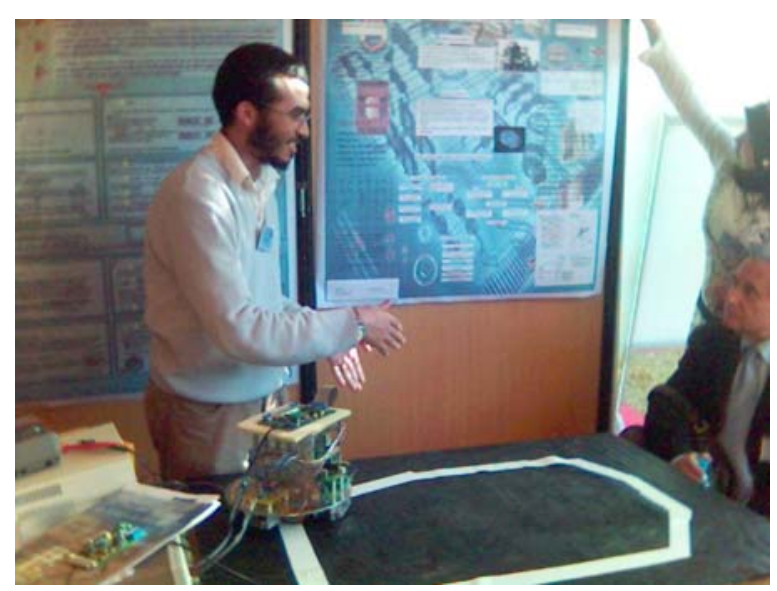

Fig. 6. Démonstration d'un suivi de ligne blanche par une plateforme robotisée

\section{CONCLUSION ET PERSPECTIVES}

De cette expérience, nous pouvons d'ores et déjà tirer plusieurs enseignements.

Tout d'abord en permettant à l'équipe enseignante marocaine de dialoguer longuement avec les concepteurs du matériel didactique qui leur était fourni, en leur permettant de participer au processus de conception, un véritable transfert de compétence a pu se faire de l'Europe vers le Maroc, avec la possibilité pour les marocains de s'approprier véritablement les outils, ce qui n'aurait jamais été possible si on leur avait fourni du matériel commercial. Moins d'un an après leur livraison, des modifications / amélioration ont déjà été apportées au plates-formes livrées, à l'initiative même de leurs nouveaux propriétaires.

Du coté des étudiants, on peut constater que, quelques semaines seulement après le début du projet, ils étaient déjà en mesure d'intégrer l'ensemble des composants au sein du robot. Cela montre que les étudiants choisis pour mener cette première expérience, maîtrisaient déjà très bien, grâce aux travaux pratiques organisés les semestres précédents, le fonctionnement des différentes parties du robot, mais que de plus, ils avaient déjà commencé à régler les problèmes d'interfaçage des capteurs avec le microcontrôleur, de couplage par les alimentations des différentes parties du système,... Bref, les étudiants semblaient déjà en bonne voie pour relever le défi de robotique mobile qui leur était posé.

Il faut dire que leur motivation vis-à-vis du projet est extrêmement élevée. Ils ont visiblement plaisir à travailler (ils avouent que c'est d'ailleurs la perspective du projet qui les a attiré vers la filière génie électrique) et ne comptaient pas leurs heures. Dans ces conditions qu'ils apprennent beaucoup en électronique de signal et de puissance, en électrotechnique, en automatique, en informatique industrielle à travers le projet, n'est pas forcément étonnant. 
A leur aisance à manipuler le système, il est par ailleurs évident que les étudiants en question ont d'ores et déjà acquis le sens pratique, la capacité à manipuler, compétences dont l'acquisition constituaient justement l'objectif du projet.

Du coté européen, ce dialogue avec leurs collègues marocains a permis aux enseignants belges notamment de retravailler le dispositif pédagogique d'apprentissage par projet en mécatronique. Pour aider les étudiants à spécifier de façon plus pertinente et plus précise les besoins en capteurs et en motorisation, des travaux pratiques longs ont été mis en place autour de plates-formes robotiques identiques à celles fabriquées pour les marocains.

Si cette approche "sur mesure" s'est avérée extrêmement positive pour les différentes parties (transfert effectif de savoir-faire vers les enseignants marocains, mise en place d'un dispositif pédagogique adapté au besoins locaux et même des retombées positives sur l'enseignement en Belgique), il faut bien être conscient de son coût, sans commune mesure avec celui du matériel finalement livré.

Quatre missions d'une semaine.homme ont été organisée pendant la phase de préparation / évaluation des besoins / présentation aux enseignants marocains des savoir-faire des partenaires européens (coût : 6k€ de frais de voyage et de séjour et autant en frais de personnel).

Le conception et la fabrication des 6 plates-formes représente un coût de près de $7 \mathrm{k} €$ en terme de composants à acheter (moteurs, réducteurs, capteurs principalement) mais plus de $25 \mathrm{k} €$ en frais de personnel.

Enfin la formation des enseignants marocains a nécessité l'organisation de 8 nouvelles missions d'une semaine.homme (2 missions pour chacune des matières, électronique de puissance, capteurs, microprocesseur et 2 à la livraison des robots pour les aspects mécaniques et l'interfaçage entre les différentes parties).

\begin{tabular}{|c|c|c|c|c|}
\hline & 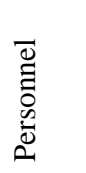 & 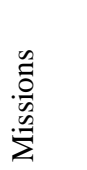 & 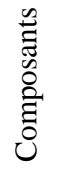 & 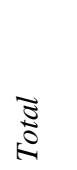 \\
\hline Préparation & $6 \mathrm{k} €$ & $6 \mathrm{k} €$ & & $12 k €$ \\
\hline Fabrication des robots & $25 \mathrm{k} €$ & & $7 \mathrm{k} €$ & $32 k €$ \\
\hline Formation des formateurs & $12 \mathrm{k} €$ & $12 \mathrm{k} €$ & & $24 k €$ \\
\hline Total & $43 k €$ & $18 \mathrm{k} €$ & $7 \mathrm{k} €$ & $68 \mathrm{k} €$ \\
\hline
\end{tabular}

\section{Remerciements}

Les auteurs tiennent à remercier la Commission Européenne pour son soutien à travers le programme Tempus-Meda. Ils associent à ces remerciements Jean-Pierre Guibergia, coordinateur de ce projet, ainsi que toutes les équipes administratives et techniques qui, au sein de l'Ecole Centrale de Marseille, de l'ENSA de Marrakech, de l'ISEN-Toulon et de l'UCL ont permis la réalisation de projet.

Ils souhaitent saisir cette occasion pour remercier tout particulièrement André Lengelé, Thierry Darras et Marie-Christine Vandigenen de l'UCL.

\section{Bibliographie}

[1] D. Grenier, S. Ivanov, F. Labrique, S. Labrique, B. Robyns. M.-J. Resende, "e-Learning en génie électrique : Développement coopératif de ressources pédagogiques multimédia", Journal de l'Enseignement des Sciences et Technologies de l'Information et des Systèmes (J3EA), Vol. 5, 21 (2006), DOI 10.151/j3ea:2006021 .

[2] D. Grenier, P. Sente, P. Fisette, B. Raucent, "La conception et la réalisation de robots mobiles comme éléments fédérateurs d'une formation en mécatronique", Actes du $2^{\text {ème }}$ Colloque sur l'Enseignement des Technologies et des Sciences de l'Information et des Systèmes (CESTIS-EEA'99), Montpellier (France), Novembre 1999, pp. 295-298.

[3] C. Vloebergh, D. Grenier, P. Fisette, C. Eugène, J.-D. Legat, "Intégration d'une compétition de robotique au sein d'un cursus de formation en mécatronique", actes $\mathrm{du}$ 5ème Colloque sur l'Enseignement des Technologies et des Sciences de l'Information et des Systèmes (CESTIS-EEA'2005), Nancy (France), Octobre 2005, papier IP-27, 6 pages (CD-ROM), repris dans Vol. 5 Hors Série 2 du Journal de l'Enseignement des Sciences et Technologies de l'Information et des Systèmes (J3EA).

[4] L. De Vroey, F. Vrins, F. Labrique, C. Trullemans, C. Eugène, D. Grenier, "Appren-tissage par projet en électricité : exemple et mise en œuvre", Journal de l'Enseignement des Sciences et Technologies de l'Information et des Systèmes (J3EA), Vol. 5, 20 (2006), DOI 10.151/j3ea:2006020

Tab. 1. Détail des coûts du projet 